\title{
LOS RICERCARE DE LAÚD O VIHUELA
}

\author{
POR ESTHER MORales Cañadas
}

\begin{abstract}
En el presente artículo se realiza una aproximación a la forma musical Ricercare, surgida a partir de las improvisaciones para danzas a proncipios del siglo XVI. Se han estudiado sus fuentes y autores más representativos, dentro de la variedad de tipologías que éstos aportaron a lo largo del siglo.

In this article, an approach to the musical form Ricercare is made, arisen from the extemporizations for dances at the beggining of the 16 th century. Its most representative sources and authors have been studied, within the variety of typologies that they contributed all along the century.
\end{abstract}

\section{SIGNIFICADO ETIMOLÓGICO}

La palabra italiana ricercare ("buscar", "probar") se utiliza en música para referirse a una forma instrumental basada en el mismo principio que la "fantasía", ambas de la misma época. Su característica principal fue, originalmente, su fin didáctico; para ello se inventó un método de libre improvisación, aprovechando todos los medios técnicos del laúd, instrumento para el que fue concebido y que produjo numerosas obras.

\section{LA MÚSICA DE DANZA COMO ORIGEN DEL RICERCARE}

Las primeras composicones que aparecieron con el nombre de ricercare fueron editadas por el italiano Ottaviano Petrucci. Se trata de un gran Opus repartido en seis tomos:

- Intabulatura de Lauto. Libro Primo (Spinacino). Venecia, 1507.

- Intabulatura de Lauto. Libro Secondo. Venecia, 1507.

- Intabulatura de Lauto. Libro Terzo. (Desaparecido).

- Intabulatura de Lauto. Libro Quarto. (Joanambrosio Dalza). Venecia, 1508.

- Tenori e contrabassi intabulati col sopran in canto figurato, per cantar e sonar col lauto. Libro Primo. (Francisco Bossinensis) Venecia, 1509.

- Tenori e contrabassi. Libro Sicondo. (Francisco Bossinensis). Fossombrone, 1511. 
De estos tomos, el Libro Terzo y el segundo de Bossinensis se dieron por perdidos, apareciendo este último hace algunos años.

Es en el libro de Joanambrosio Dalza, o sea, en el tomo Intabulatura de Lauto, Libro Quarto de la edición de Petrucci, donde se encuentra la fuente principal para la forma Ricercare e incluso para la música de laúd de la época. En este libro encontramos un sinfín de tocatas -bajo el nombre de Tastar de cordas y Ricercare-, así como innumerables danzas y algunos arreglos para laúd de composiciones vocales.

Dalza es, pues, el primero que en su obra habla de una maniera de cómo hay que descifrar la tabulatura de laúd, dando al mismo tiempo una explicación de la sucesión de danzas. Así se puede leer en el primer folio: "Nota che tutte la pauana hanno al suo saltarello e piva." ("Ha de tenerse en cuenta que a cada pavana le sigue su saltarello y su piva"). Y continúa después en el mismo folio: "Tastar de cordas con li soi ricercar drieto" ("Tocata con su sucesivo ricercar").

Por todo ello, así como por su introducción y resumen, ha de considerarse la colección de Dalza como la primera tabulatura de laúd que explica claramente no sólo la sucesión de danzas, sino también la asociación entre Tocata y Ricercare.

Es obvio, sin embargo, que para comprender la función del Ricercare hay que tratar en primer lugar las danzas en sí mismas. Se puede decir que la música de danza durante el siglo XV es, en cierto modo, la mediación o el enlace entre la música no escrita -toda aquella que era improvisada- y la forma idiomática de escribir la música culta instrumental. Exceptuando algunos casos, se debe considerar que toda la música de danza dependía casi exclusivamente de los libros de enseñanza de la misma, los cuales eran la base para una coreografía escrita determinada, que a su vez se apoyaba en una voz tenor con valores "breves" -o sea, sin ritmo indicado-, tal como aparece en el Manuscrito Burgundio de Margarita de Austria, de la Biblioteca Real de Bruselas -Ms. 9085-. En otros casos podía formarse sobre una melodía mensural, como ocurre en los escritos de Piacenza y sus seguidores, Guglielmo Ebreo y Antonio Comazano.

Con la aparición de la imprenta musical se fomentó el interés por la música instrumental, a la que llevó más allá de las fronteras que hasta ahora había alcanzado. Y así, si hasta este momento la música había sido algo que sólo estaba en manos de los profesionales, con la impresión de notas iba a pasar a estar al alcance de todos, fueran músicos o no, como un medio social y recreativo. $Y$ precisamente fue con este fin con el que Petrucci, en 1501 dio a luz su Odhecaton, colección de obras musicales vocales e instrumentales, con o sin textos. A estas piezas les siguieron otras genuinas para el laúd, pero sobre todo piezas de danza, hechas de forma simple tanto armónica como formalmente, las cuales llegaron a formar parte de la "música ligera" de la época.

Volviendo a Dalza, él menciona en su libro tres tipos de danza: Pavana, Saltarello y Piva. No obstante, una explicación más amplia se encuentra ya en 
Cornazano, en su Libro dell'arte de danzare '. Cornazano describe exactamente las formas de improvisación a las que han de atenerse la tocatas para poder dar la métrica determinada a las diferentes danzas. Esta métrica se encuentra siempre en la voz del tenor para las llamadas "danzas bajas" -Basse-Dance-, las mejores según Cornazano. Y así, a partir de los diferentes compases distingue Cornazano cuatro tipos de danza:

1.- Danza que contiene tres pasos (Botte) para cada nota del tenor. A esto se le llama en Italia Saltarello.

2.- Danza Quaternaria. Está formada por cuatro Botte para cada nota. Es la más utilizada para las Allemandas.

3.- La Cacciata, que es igual a la Piva. También se le llama Figliola de la Quaternaria (hija de la cuaternaria), porque consta igualmente de cuatro pasos para cada nota, aunque a doble velocidad.

4.- La Basse-Dance o Misura Imperiale. En ella se dobla el valor de cada nota, es decir, tres se valoran como seis, seis como doce, etc. ${ }^{2}$

Como se puede deducir, la descripción de estas danzas -Quaternaria, Saltarello y Piva- demuestra que son en sí improvisaciones sobre un cantus firmus en la voz del tenor. Y si se compara el ritmo de las danzas de Cornazano con el de las de Dalza, se llega a la conclusión de que son exactas. (Ejemplo 1)

Lo esencial de estas variaciones es la utilización de figuraciones que se suceden por grados conjuntos sobre una nota fundamental en la voz del tenor. Se puede considerar como una técnica de "disminución", una especie de variaciones a través de ornamentos. Un ejemplo de ello se puede ver en el Saltarello para dos laúdes de Dalza (ej. 2). Esta composición es en sí bastante primitiva y supone realmente sólo una disminución melódica sobre un Fauxbordon o un bajo ostinato. No obstante, se pueden sacar de aquí las características del método de improvisación de Dalza, y éstas son las que siguen:

1.- La disminución transcurre en grados conjuntos.

2.- Utilización de cadenas de mordentes de tres notas.

3.- Conexión entre los acordes de terceras, sextas y décimas.

Hay que decir, debido a la pobreza económica de estas danzas para laúd, que son la muestra de que fueron escritas para la utilización práctica de los no profesionales, sobre todo si se las compara con los arreglos de obras vocales, y por supuesto con los Ricercare -llamados también Fantasías, Preambel o Priamell-, de una mayor riqueza y fuerza rítmica que los anteriores.

1. Codex Capponiano, n.203. Bib. Vaticana. Del 1455, 14656 1495-90.

2. Mazzi, C.: Il libro dell'arte de danzare. A. Cornazano in La Bibliofilia. Florencia, 1916. Anno $12, \mathrm{n}^{\circ} 1$. 


\section{Los Ricercare de J.A. Dalza}

Los Ricercare de Joanambrosio Dalza muestran la misma técnica improvisadora de las danzas, y esto se puede comprobar al comparar dos incipit: el del Ricercare $\mathrm{N}^{\circ} 7 \mathrm{~b}$ y el de la Pavana alla Venetiana (ej. 3). Por supuesto tienen su diferencias, y éstas se encuentran en la asimetría de la forma -ya que las danzas no están conectadas a la voz del tenor-, así como también en su relación con la tonalidad: aunque las danzas no tienen tonalidades bien definidas, se encuentran agrupadas según éstas; los Ricercare en cambio muestran una gran inestabilidad tonal, de tal modo que algunos comienzan en do menor y terminan, por ejemplo, en Fa Mayor -como es el caso del Ricercare $\mathrm{N}^{\circ} 12-$, y otros se alejen tanto de la tonalidad que su Tocata sucedánea ya tiene otra totalmente distinta -como el Ricercare $\mathrm{N}^{\circ} 45-$. Aunque también hay excepciones, en las que se mantiene la tonalidad casi desde el comienzo hasta el fin.

Aparte de todo esto, los Ricercare son piezas dependientes de otras que los preceden y los prosiguen, y están adaptados, por así decirlo, a ellas. Es más, la característica de estas composiciones está basada en el anudar de un motivo que se desarrollará a través de todas las voces, pero sin una construcción periódica o articulada y sin continuidad tonal, tan sólo con cadencias finales formadas por progresiones que dan pie a un final muy efectista. Sin embargo es más relleno que verdadero sentido compositivo. Ejemplo de ello se puede ver en el siguiente Ricercare de Spinacino, que utiliza la misma técnica que Dalza (ej. 4) ${ }^{3}$

Los Ricercare no se suelen encontrar situados siempre ene el mismo lugar. Pueden aparecer tanto como Preludios, Interludios o Postludios, según se los coloque al principio, en el medio o al final de las Suites. Pueden formar también pareja con las Tocatas, cumpliendo éstas en tal caso una doble función:

1.- Determinación de la tonalidad. Aunque la tonalidadesté determinada por el Ricercare, es la Tocata, que lo antecede, la que realmente la determina, como una intonazione; es decir, la Tocata de el tono al cantante, pero sólo con acordes. Estos acordes pueden estar unidos armónicamente entre sí, pero aparecen aislados y sirven simplemente de colorido para el tono.

2.- Como obra principal. Los Ricercare actúan aquí como Coda de la Tocata, la cual se ajusta, también en este caso, a la tonalidad de aquellos, por ser de mayores dimensiones. Ésta es en realidad la forma típica del siglo XVI y la que en J.A. Dalza aparece con el nombre de Tastar de cordes. ${ }^{4}$

El Tastar de cordes es una pieza de 2/4 con muy pocos elementos temáticos, que se desarrolla sin una periodicidad y con poca tendencia a modular, con sólo figuraciones de notas blancas que se suceden unas a otras, simplemente sostenidas

3. PETRUCCI, Libro I, Pág. 39; en JACQUOT, Jean: Le Luth et sa Musique (Recopil.), París, 1980.

4. Die Ältesten Denkmaler der Orgelmusik als Beitrag zur Geschichte. Leipzig, 1927. 
o bien haciendo coloraturas. Y esto es lo que las diferencia de su Ricercare, el cual, tomando la idea de su Tastar y sin separarse de él, se ensancha rítmicamente hasta el final (Ej. 5).

Resumiendo, el Ricercare era sólo un modelo de improvisación para que cualquiera pudiera tocar el laúd, aunque para ello fuese necesario un mínimo de conocimiento de la tabulatura y de dominio del instrumento. Tienen evidentemente un fin didáctico, especialmente para aficionados, ya que tanto Dalza como Spinacino contaban con poca clientela bien formada profesionalmente; muchos de ellos no sabían ni siquiera leer música, ni mucho menos latín, idioma en el que se escribían entonces las obras teóricas. Así escribe Spinacino el título de su colección en latín e italiano: Regula pro illis qui canere nesciunt y Regola per quelli che non sanno cantare ("Regla para aquellos que no saben leer notas"). Y a continuación sigue un tabla de improvisaciones o Ricercare en todas las tonalidades (ej. 6).

Hay, sin embargo, una colección que se considera como la más antigua de todas las referentes al laúd. Se trata de composiciones de contemporáneos de Dalza que también escribieron para el laúd, bien sobre una voz tenor, bien sobre melodías de Madrigales o Chanssons. La mayoría de los compositores son anónimos, pero también aparecen algunas obras de Sartori. Todo esto lo menciona G. Thibault en su estudio Le luth et sa musique. Un manuscript du XVI siécle ${ }^{5}$. Thibault reconoce este manuscrito como la fuente más importante para la música de laúd, entre otras cosas porque no tiene fines didácticos, como ocurría con los libros de Petrucci e incluso con los de Capirola de 1517 (ej. 7). Son pues piezas de profesionales y para profesionales, y ha de poseerse una determinada técnica lautista para dominarlas.

El manuscrito se compone, entre otras, de Frottolas, Calatas, Ricercare y algunos Motetes, piezas éstas que, según transcripciones posteriores, hacen datarlo hacia 1480 , al menos como punto de partida.

No obstante, este manuscrito es una expcepción, pues todos los posteriores, ya sea en Italia como en Alemania, fueron escritos con ese fin pedagógico, como lo demuestra igualmente la obra de Hansen Neusedles, en Alemania, en cuyo preámbulo se lee:

"Ein sehr kunstreicher Preambel oder Fantasy, darin sind begriffen vil mencherley art von zwiefachen und drifachen doppel laiffen, auch sincopationes und vil schöner fugen, durch mich Hansen Neusedle lutenisten zu zamen gepracht und corrigiert". ${ }^{6}$

Esta obra se puede considerar como un auténtico catálogo de todos los recursos posibles para la improvisación, desde el punto de vista pedagógico. En

5. MURPHY, Richard, "Fantasie et Ricercare"; en JACQUOT, op. cit.

6. "Ein neugeordnet künstlich Lautenbuch", citado en Ernst POHLMAN, Laute, Theorba, Chitarrone. Die Instrumente, ihre Musik un Literatur von 1500 bis zur Gegenwart. Bremen, 1982. 
ella se muestran todos los modelos posibles: posición de acordes, pasajes por grados conjuntos o disminuciones, ornamentos de mordentes de tres notas, pasajes que forman un diálogo, terceras y sextas paralelas, progresiones, contrapunto simulado, etc.

\section{Desarrollo del Ricercare}

En la segunda mitad del siglo XVI alcanzó el Ricercare un gran significado y al mismo tiempo se fue renovando en su estilo formal. La colección de Capirola, editada en 1517 , era ya muy conocida.

En esta época comienza al cultivarse el Ricercare de imitación. El primero de esta especie apareció editado en la colección Musica nuova acomodata per cantar e sonar sopra organi, et altri strumenti (1540). Dicha colección contiene piezas de Giulio da Modena, A. Villaert y otros. ${ }^{7}$ Muy poco después se publicaron los primeros Ricercare imitativos para órgano de G. Cabazzoni (1543).

Igualmente, aunque un poco más lentamente, el Ricercare de laúd se acercará a la forma imitativa impuesta por el de órgano, así surgieron los primeros con Domenico Bianchini (1546), Francesco da Milano (1547), S. Gintzler (1547), B. Baltark (1552); y en España con los Tientos -que son, junto con la Fantasía, la correspondencia semántica de estas piezas- de Manuel de Fuenllana y Luis Milán (1554) y otros muchos.

En general se puede decir que estos Ricercare tienen ya un sentido más serio y profesional que los primeros: se dividen en varios episodios, en cada uno de los cuales se trabajan, a modo de imitación, diferentes sujetos ("soggeti"). Más tarde se trabajará contrapuntísticamente un sólo soggeto, lo cual lo acercará al estilo de las Canzoni; y es que, en realidad, los temas de los Ricercare estaban muy a menudo sacados de Canzoni y de Madrigales.

Con Francesco da Milano, llamado "el divino", aumentó el interés por estas estructuras polifónicas, no sólo para las composiciones de órgano, sino también para las de laúd. Francesco da Milano no era sólo tocador de laúd, sino también organista, y admiraba la música de Josquin Desprez. Por ello tuvo la idea de trasponer varias Chanssons de este compositor, así como madrigales italianos y motetes latinos para el laúd, lo cual implicaba una determinada técnica y dominio del instrumento que hicieran posible la realización práctica que exige la polifonía.

También en España vieron la luz importantes obras y ediciones. Luis Milán publicó en 1536 su obra Libro de Música, intitulado "El Maestro", el que trata el mesmo estilo y orden/ que un maestro trataría con un principiante:/ mostrándole ordenadamen/ te desde los principios toda cosa quel podría ignorar, para entender la/ presente obra. Esta obra contiene, aparte de composiciones para canto y vihuela y algunas pavanas, una gran cantidad de fantasías, que son, junto con los tientos, los ricercare españoles.

7. Monuments of Renaissance Music. Chicago, 1964. 
Milán distingue dos tipos de fantasías/ricercare:

1.- Fantasía en consonancia con Redoble.

2.- Fantasía de imitación.

La Fantasía en consonancia es un ricercare ormnamental según la antigua tradición, mientras que la de imitación es lo mismo que los llamados ricercare de imitación.

También es importante añadir, para reafirmar el carácter serio de estas composiciones, cómo describe Milán las diferentes facetas o escalones que un intérprete debe pasar para llegar que un intérprete debe pasar para llegar a tocar bien el instrumento:

1.- Técnica del instrumento.

2.- Los modos y las cadencias.

3.- Consonancias y ornamentación.

4.- Proporciones rítmicas, etc., así como todos los elementos imprescindibles para la buena interpretación de las Fantasías.

Esto demuestra una vez más que, aunque el ricercare se haya hecho más serio y profesional, no deja de tener implícito ese carácter didáctico de su origen. Sin embargo, más importante que esto es el hecho de que los ricercare de imitación contribuyeron al mismo tiempo al desarrollo de un método de improvisación. Este método se inspiró primero en la técnica de la polifonía, para luego imitarla. Se podría considerar como la primera puerta de la improvisación para instrumentos de cuerda y tecla, y por ello encontramos que las obras de este tiempo están escritas indistintamente para ambas familias de instrumentos.

La aclaración y la explicación de todos estos métodos se encuentran en un libro clave para toda la música de la época, e incluso de épocas posteriores: $E l$ arte de tañer fantasía (Valladolid, 1565) de Fray Tomás de Santa-María. 


\section{NOTA A LOS EJEMPLOS MUSICALES:}

Los ejemplos $n^{\circ} 1,2,3,5$ y 6 son extraídos del libro de OSWALD KÜRTE, Laute und Lautemusik bis zur Mitte des 16 Jahrhunderts, Leipzig, 1901.

Los ejemplos 4 y 7 proceden de R. MURPHY, "Fantasie et Ricercare"; en JACQUOT, op. cit.

\section{Ejemplo 1}
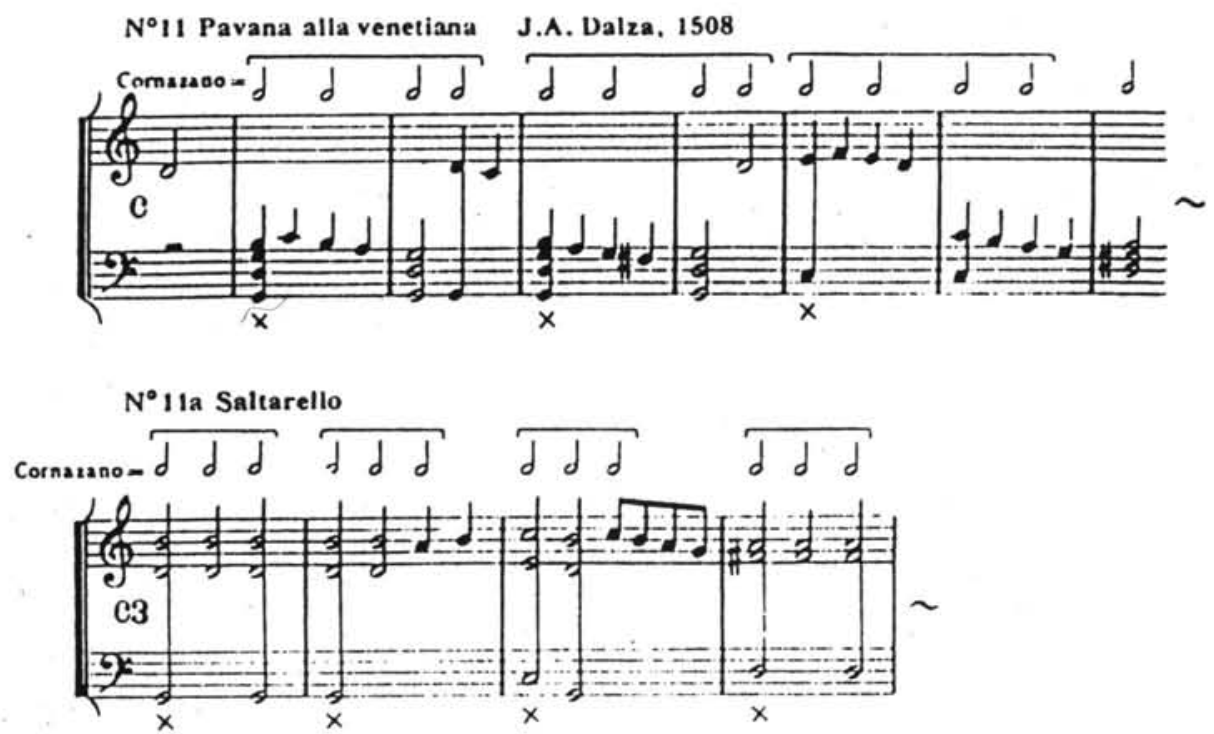

Ejemplo 2

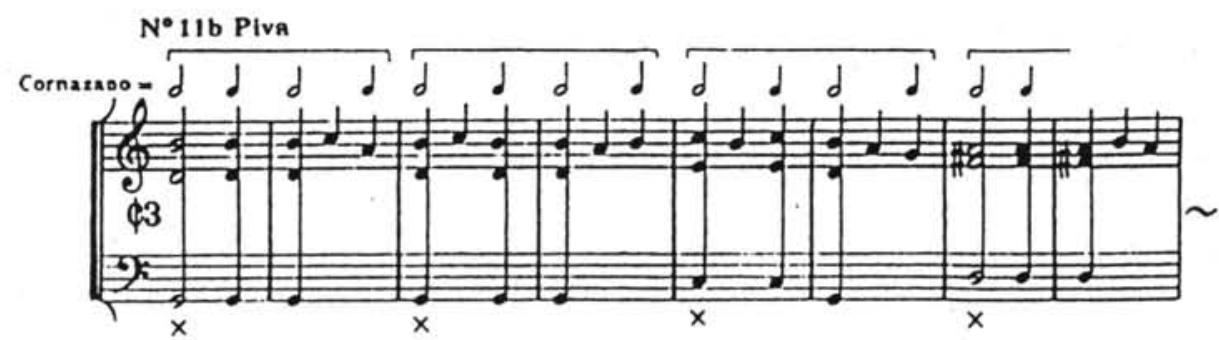


Ejemplo 3
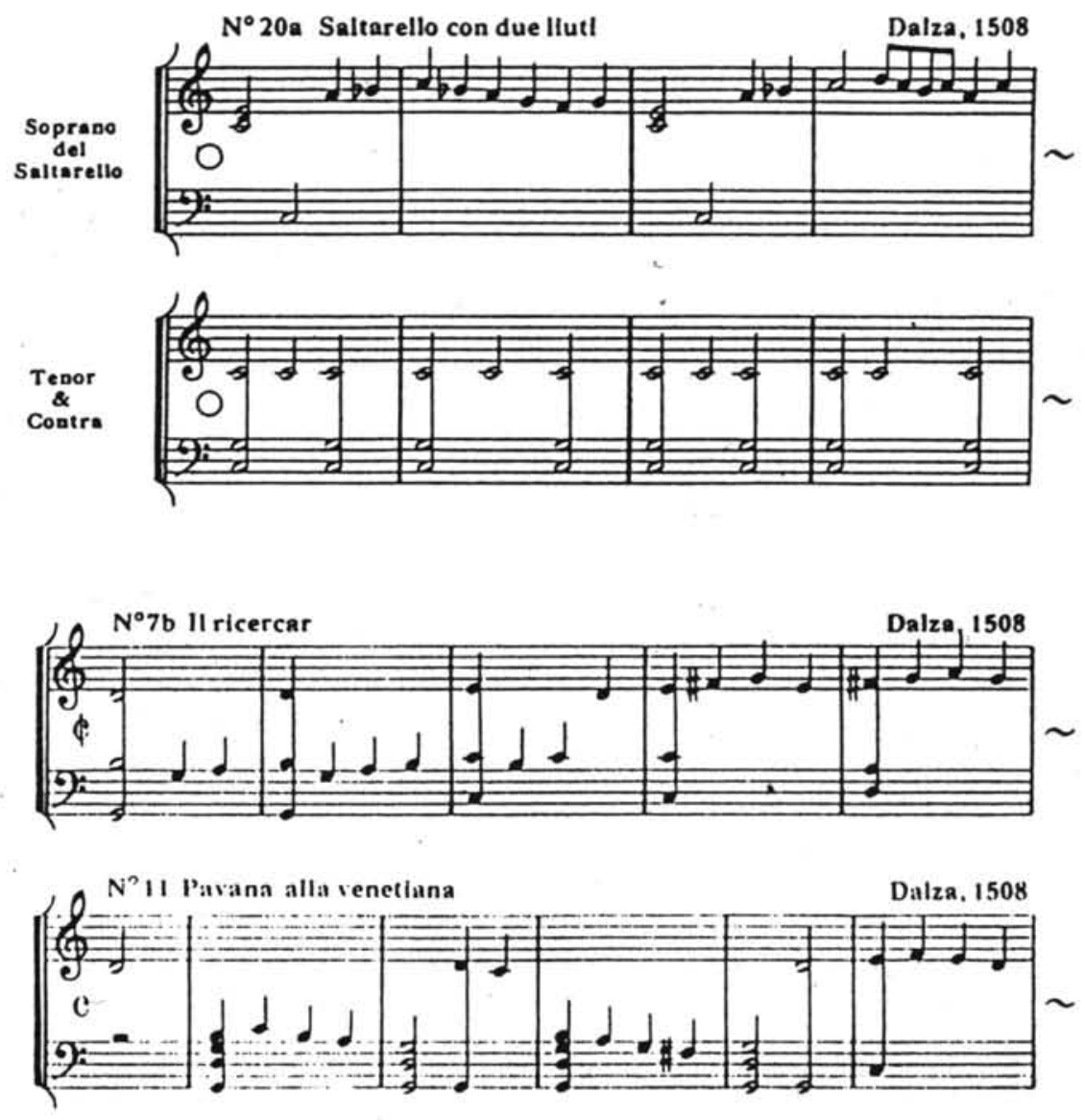
Ejemplo 4

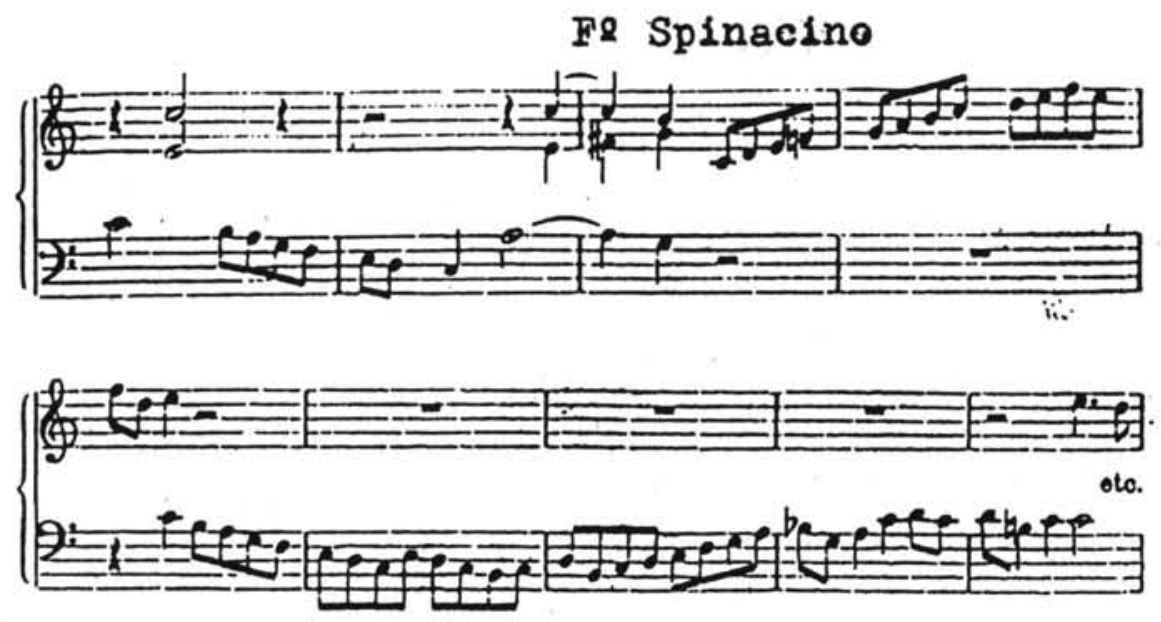

Ejemplo 5

Petrucci, Libro IV, 1508.

Tastar de corde mit Receroar (dietro). Dalza

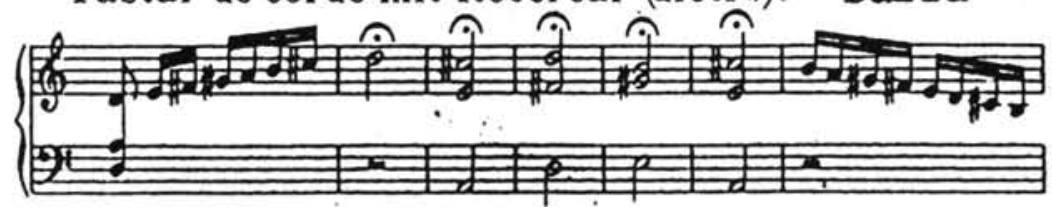

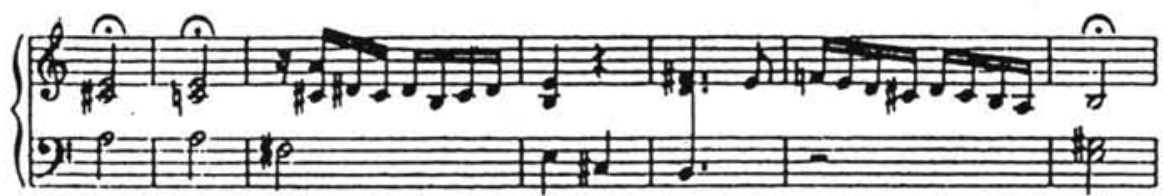


Ejemplo 5 (cont.)
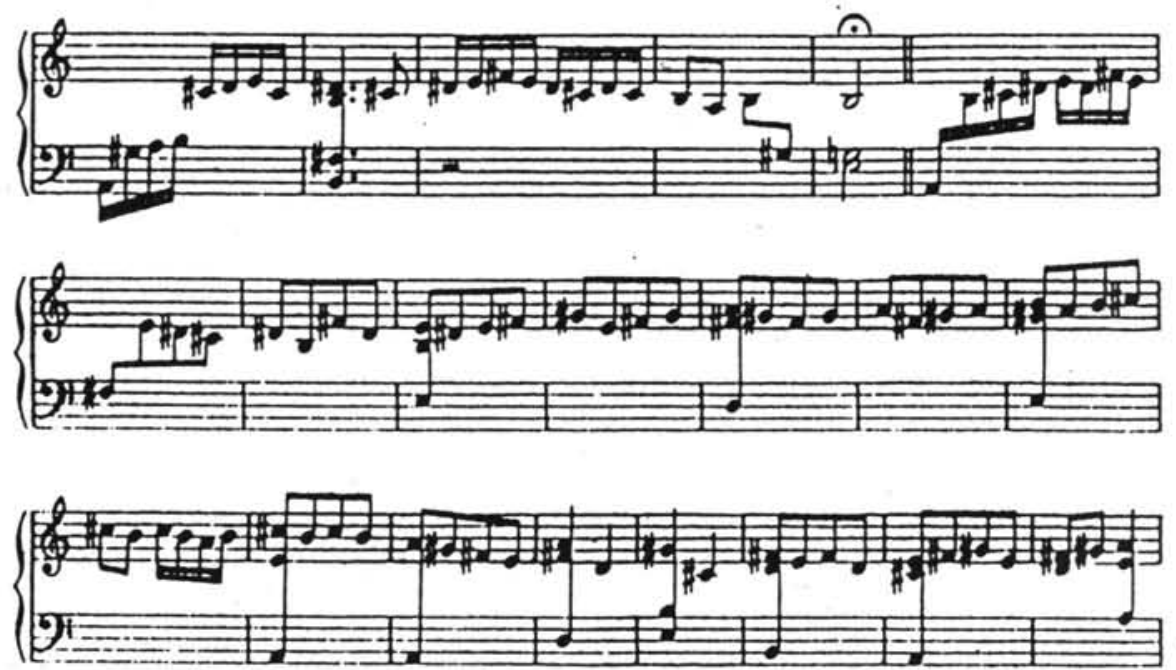

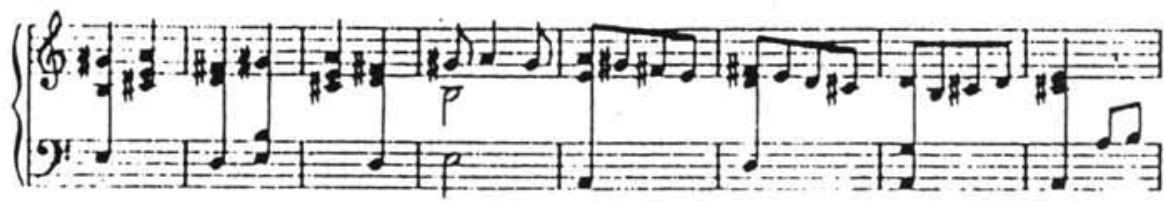

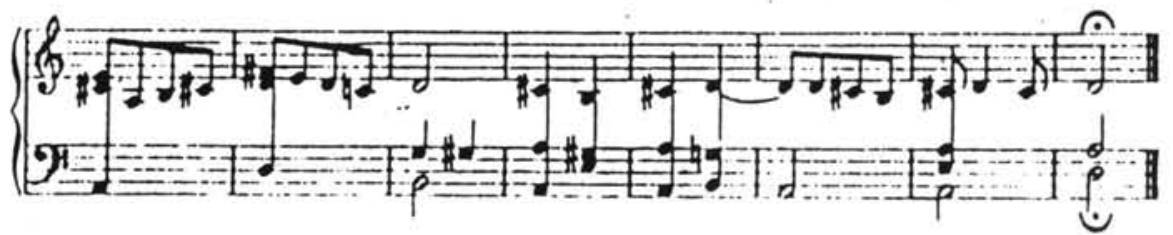




\section{Ejemplo 6}

Petrucci, Libro I, 1507.
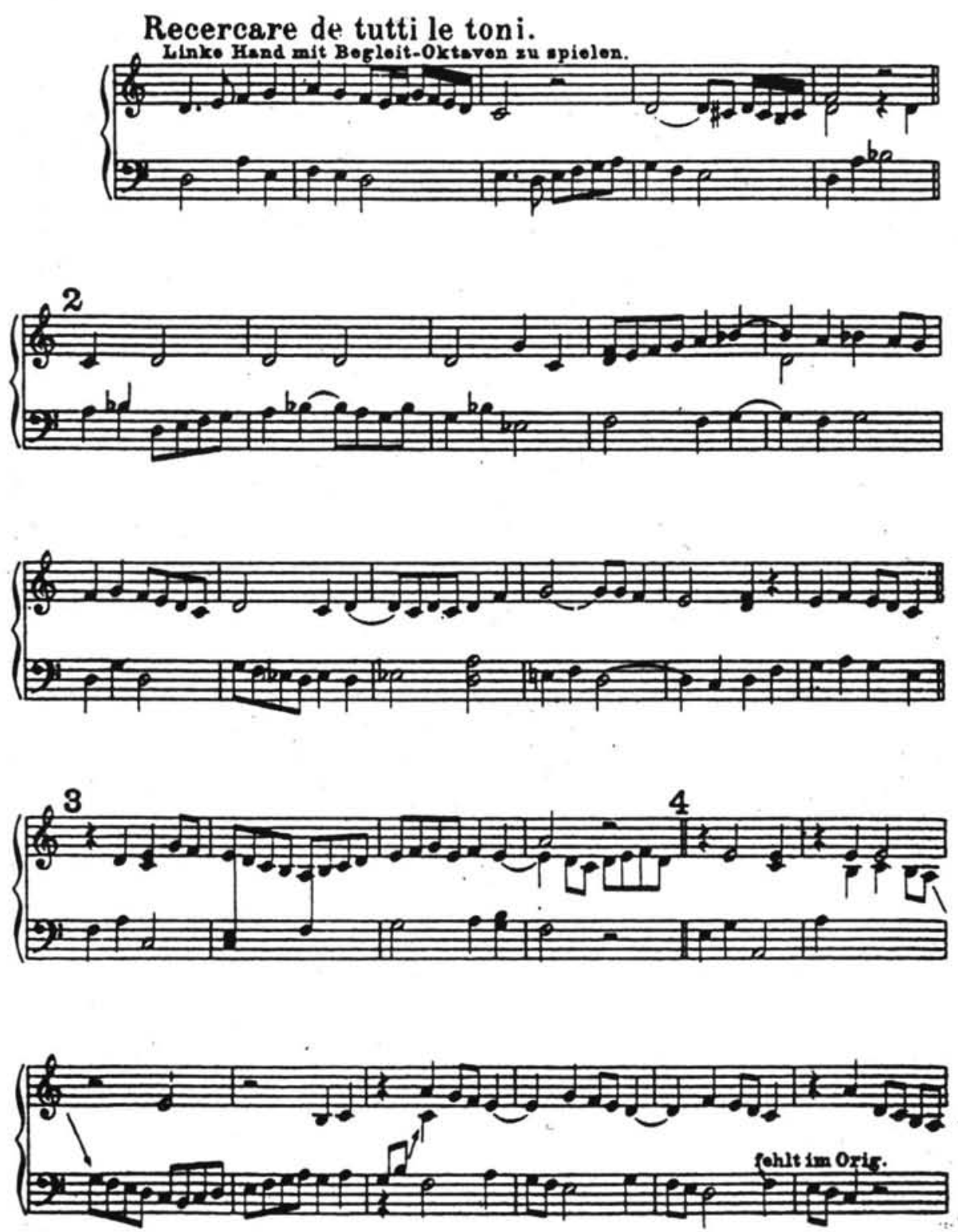
Ejemplo 6 (cont.)
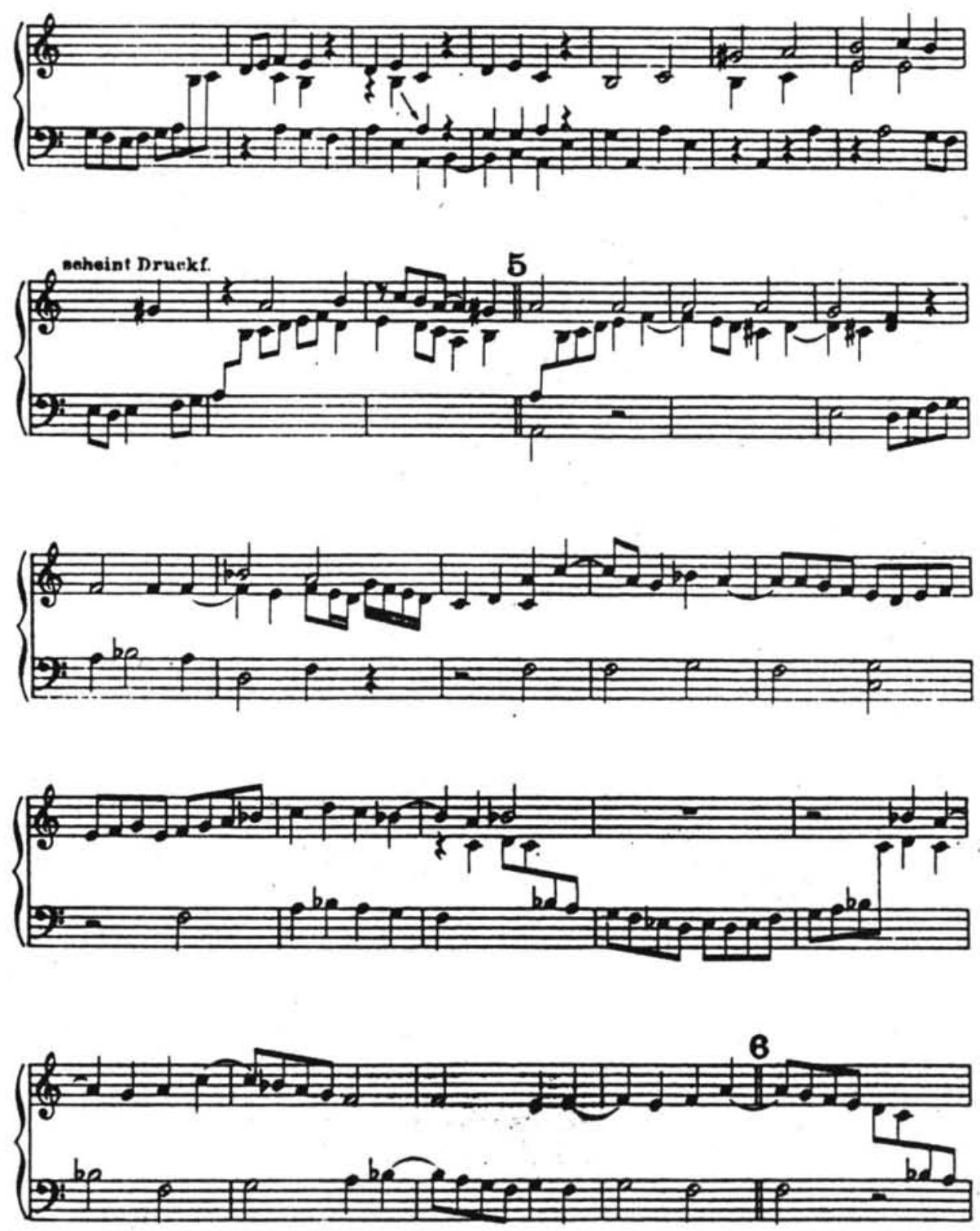
Ejemplo 6 (cont.)
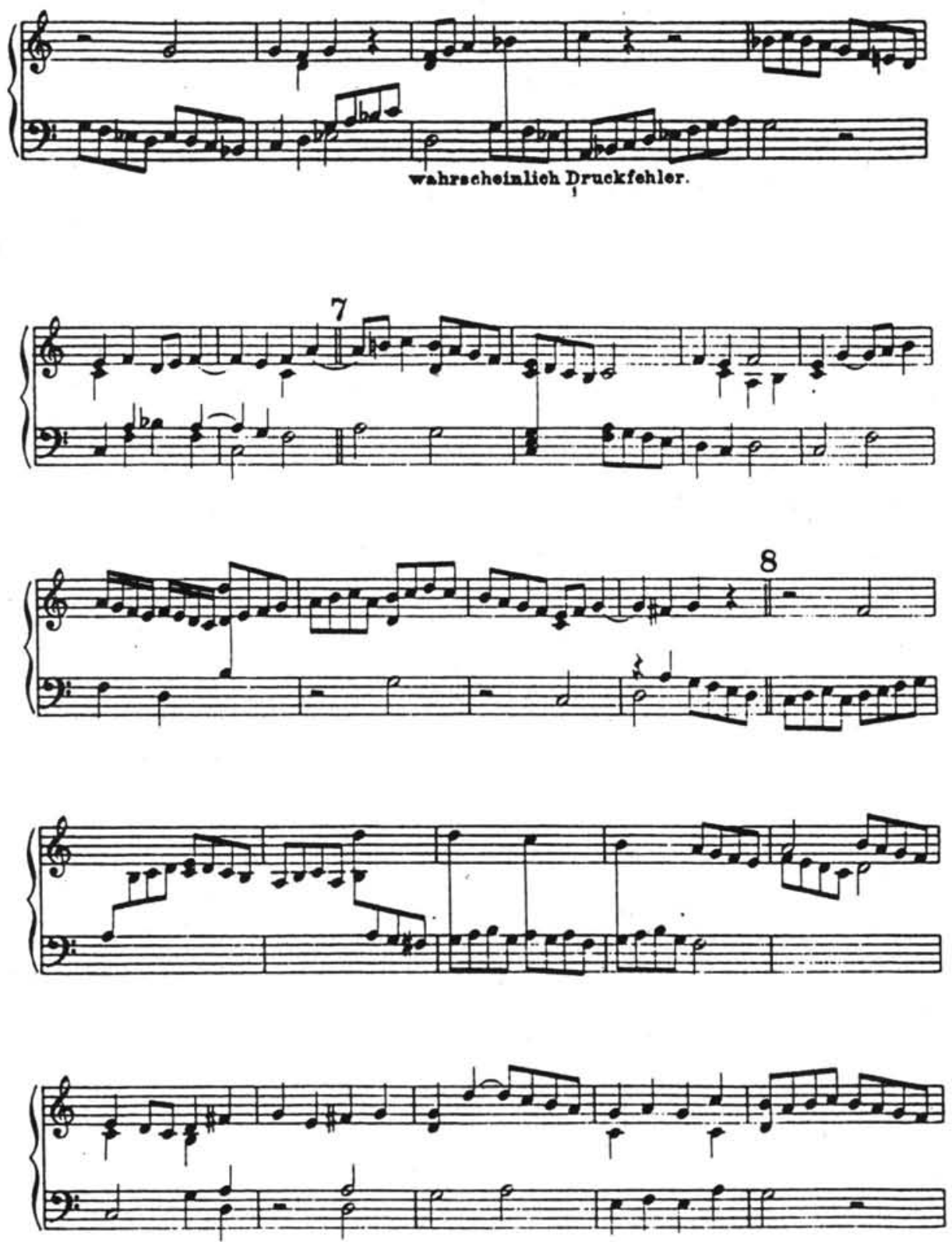
Ejemplo 6 (cont.)
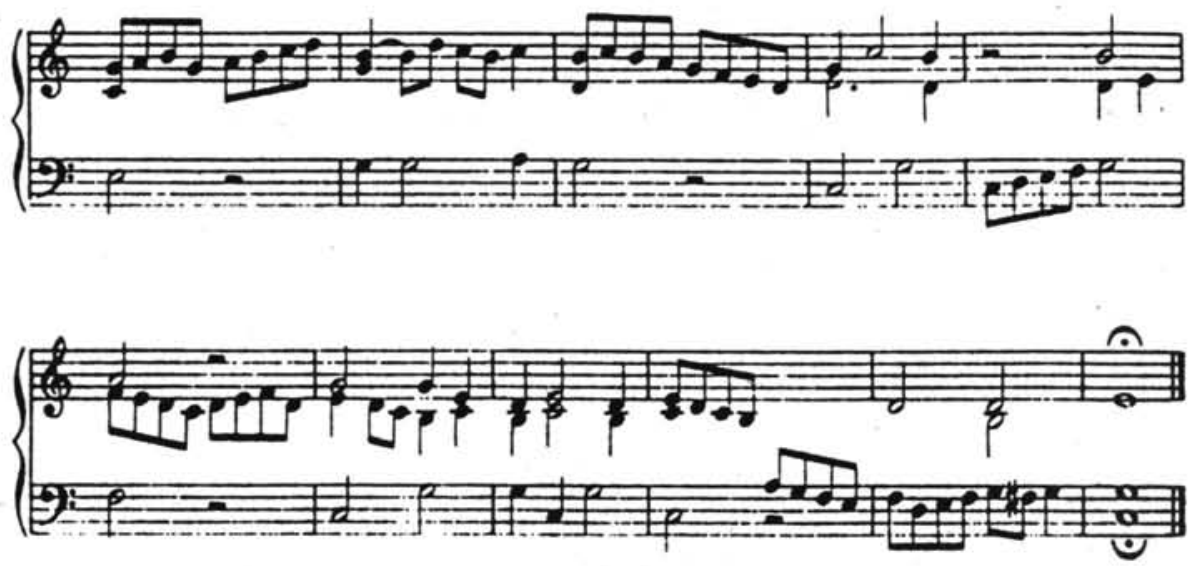


\section{Ejemplo 7}

Ricercar VI - Tablature de Capirola

(transcription par Otto (iomnosi)
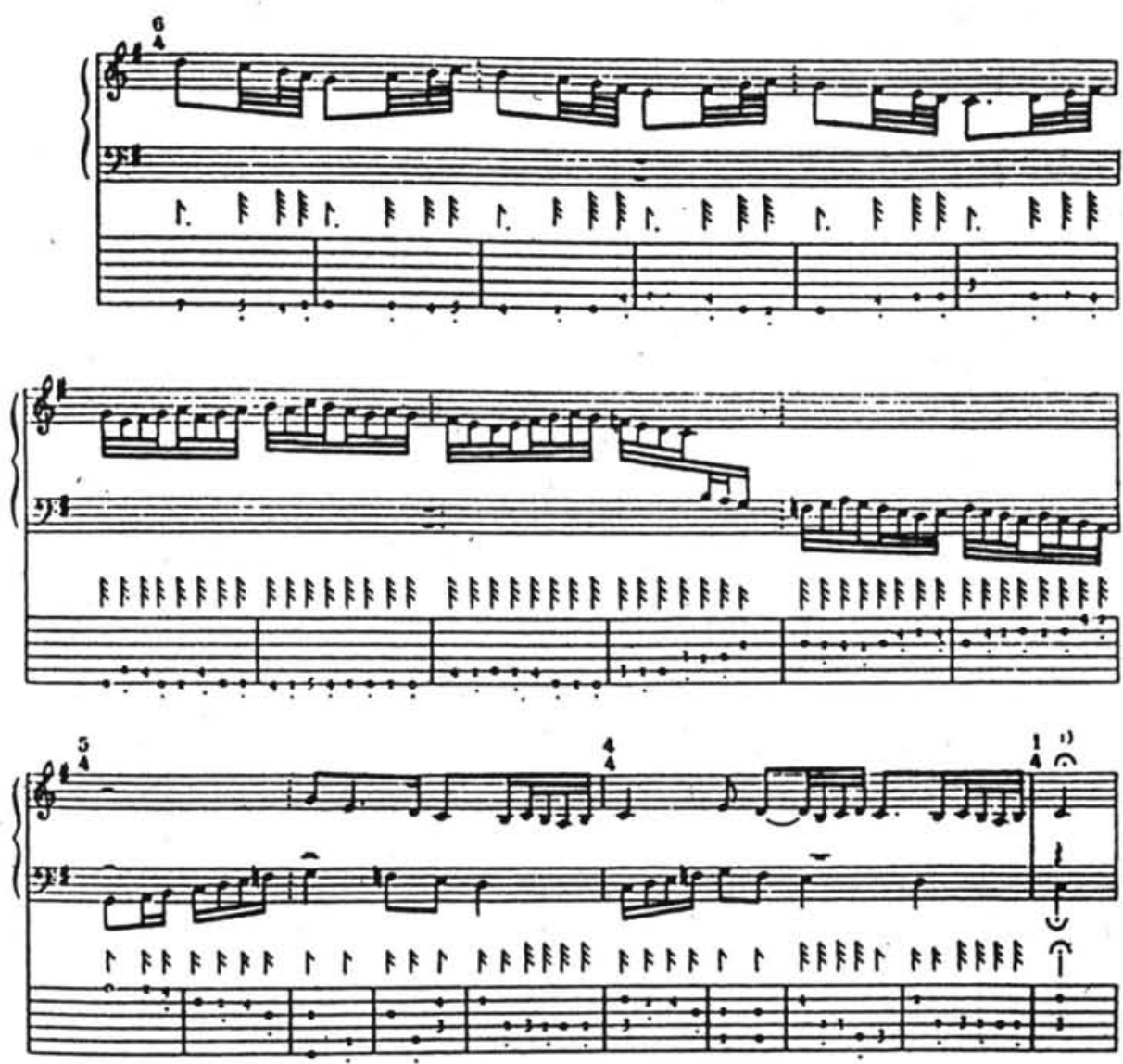